\title{
Strongyloides Hyper-Infection Syndrome and Ciliocytophthoria Mimicking Balantidium coli
}

\author{
Moni Roy ${ }^{1}$, Sharjeel Ahmad ${ }^{2}$, Arif Sarwari ${ }^{3}$ \\ ${ }^{1}$ Department of Internal Medicine, University of Illinois College of Medicine at Peoria and OSF Saint Francis Medical Center, Peoria, IL, USA \\ ${ }^{2}$ Section of Infectious Diseases, Department of Internal Medicine, University of Illinois College of Medicine at Peoria, Peoria,IL, USA \\ ${ }^{3}$ WVU School of Medicine, Department of Infectious Diseases, Morgantown, WV, USA
}

\section{Doi: 10.12890/2021_002707 - European Journal of Case Reports in Internal Medicine - ๑ EFIM 2021}

Received: 11/06/2021

Accepted: $14 / 06 / 2021$

Published: $23 / 07 / 2021$

How to cite this article: Roy M, Ahmad S, Sarwari A Strongyloides hyper-infection syndrome and ciliocytophthoria mimicking Balantidium coli. EJCRIM 2021;8: doi:10.12890/2021_002707.

Conflicts of Interests: The authors declare there are no competing interests.

This article is licensed under a Commons Attribution Non-Commercial 4.0 License

\section{ABSTRACT}

Ciliocytophthoria is a phenomenon where degenerated cells in infections or malignancy can present as ciliated cells on microscopy and so may be confused with ciliated parasitic infection. We present an interesting case of recurrent shortness of breath, misdiagnosed as chronic obstructive pulmonary disease exacerbations leading to unnecessary exposure to antimicrobials and steroids. The case was diagnosed as Strongyloides hyper-infection syndrome. Another finding worth mentioning was that ciliated cells noted on broncho-alveolar lavage were thought to be a co-infection with Balantidium coli but were later confirmed as ciliocytophthoria.

\section{LEARNING POINTS}

- Strongyloides hyper-infection syndrome should be considered in the differential diagnosis of a patient from an endemic area presenting with non-resolving respiratory symptoms.

- Ciliocytophthoria is a type of degenerative process where degenerated cells can appear ciliated on microscopy.

- Balantidium coli appears to be similar but is much larger and has cilia circumferentially compared with ciliocytophthoria which has a polar distribution of cilia.

\section{KEYWORDS}

Strongyloidiasis, hyperinfection syndrome, ciliocytophthoria

\section{CASE DESCRIPTION}

A 68-year-old woman with stable chronic lymphocytic leukaemia (CLL), hypogammaglobulinemia, chronic obstructive pulmonary disease (COPD) and hypertension had multiple admissions to a local hospital for presumed COPD exacerbations. She had received multiple courses of steroids and antibiotics over the previous 7 months. She was transferred to a tertiary care facility secondary to respiratory failure requiring ventilatory support. She did not have any known exposure to pigs or other farm animals. She did not have eosinophilia on initial presentation. A Papanicolaou (PAP) stain of the broncho-alveolar lavage (BAL) sample obtained at admission showed rhabditiform larvae of Strongyloides stercoralis (Fig. 1, Video 1) and a single ciliated respiratory epithelial cell (Fig. 2, Video 2) which was initially identified as Balantidium coli. The patient's stool specimens were also positive for Strongyloides. 

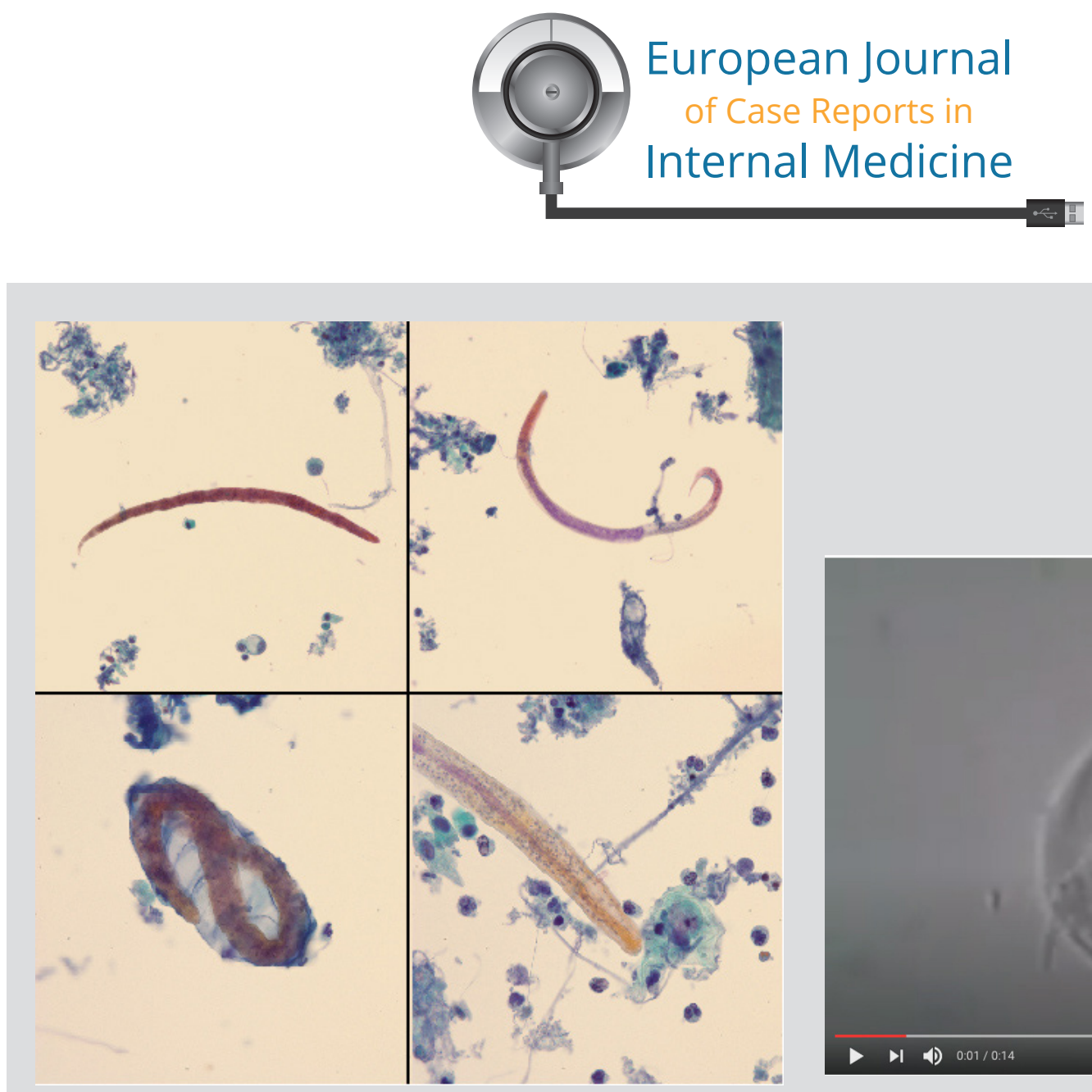

Figure 1. Pap smear of bronchoalveolar lavage specimen 1. Top right and left (200x) show rhabditiform larvae of Strongyloides stercoralis. Bottom left (500x) shows larva to be enclosed in ovum remnant. Bottom right (500x) shows a close-up view of the larva
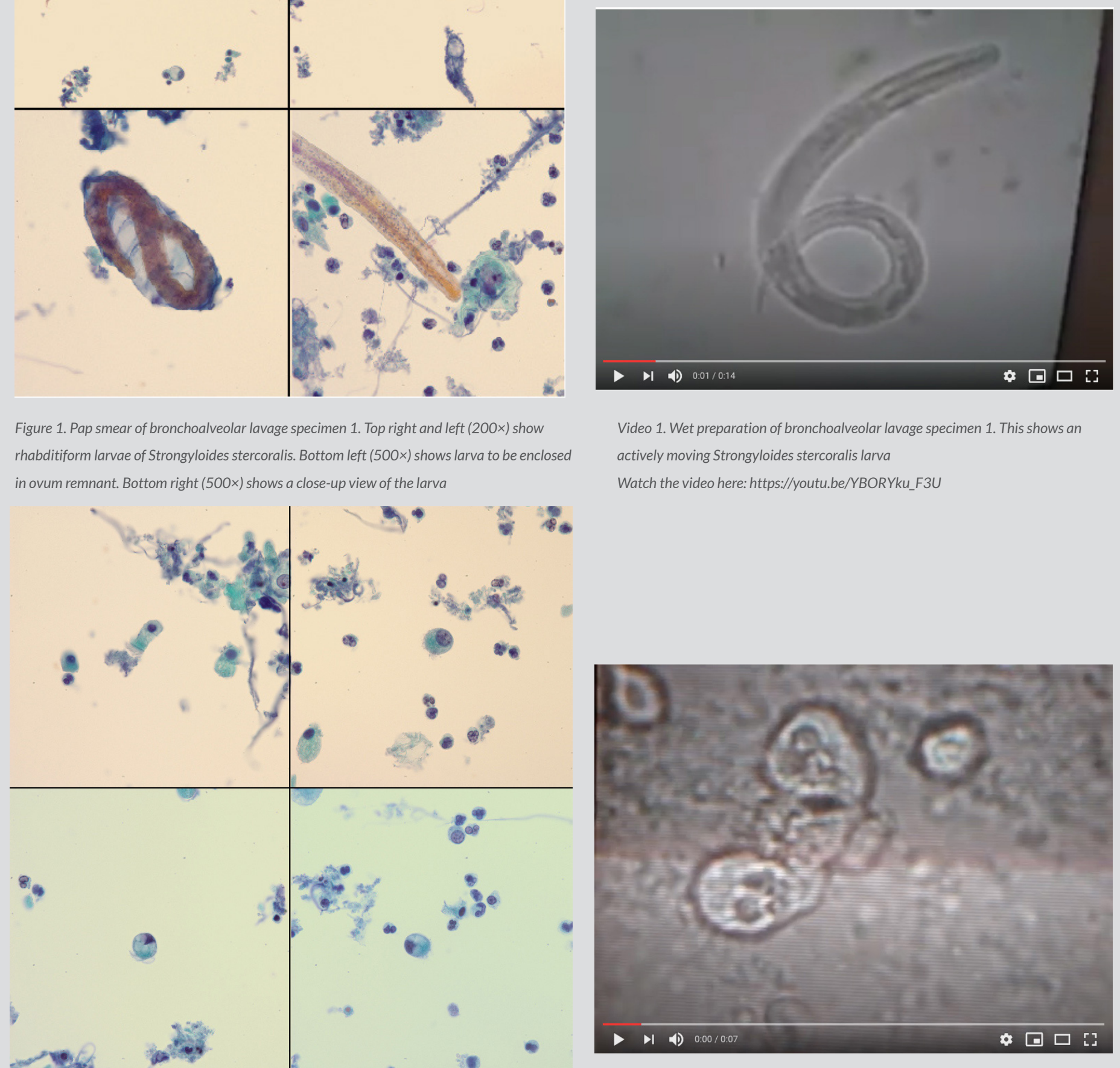

Figure 2. Pap smear of bronchoalveolar lavage specimen 2. Top left (1000x) shows a single ciliated respiratory epithelial cell. Compare this with ciliocytophthoria (CCP) shown in the top right, bottom right and bottom left panels (1000x). Note the small size and polar cilia

Video 1. Wet preparation of bronchoalveolar lavage specimen 1. This shows an actively moving Strongyloides stercoralis larva

Watch the video here: https://youtu.be/YBORYku_F3U

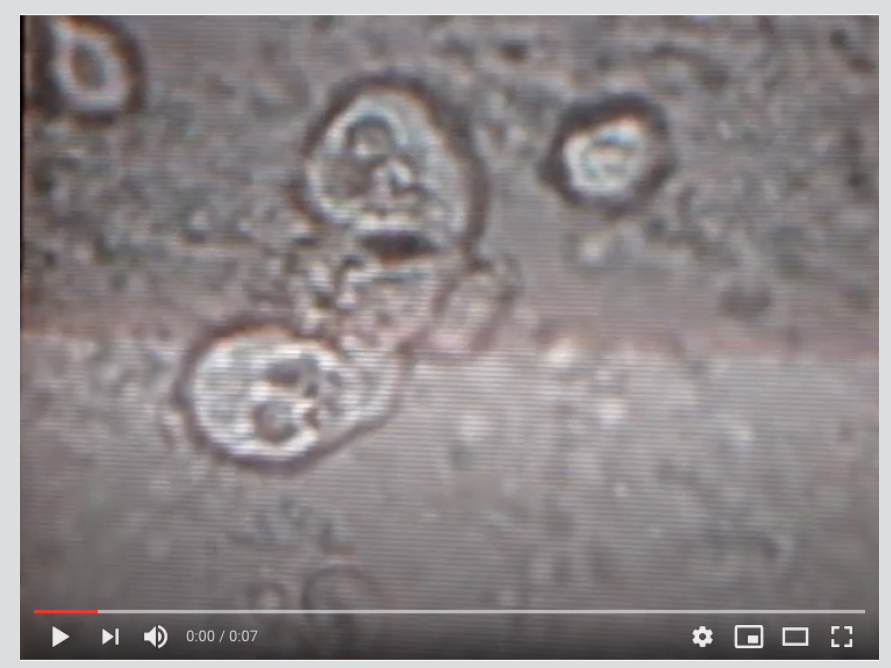

Video 2. Wet preparation of bronchoalveolar lavage specimen 2. This shows ciliocytophthoria (CCP). Note clumping of cells with polar cilia Watch the video here: $h$ ttps://youtu.be/KUWMk6KazXg 
Due to her history of hypogammaglobulinemia, the patient was treated with intravenous immunoglobulins (IVIG) in addition to a 14day course of ivermectin $(200 \mu \mathrm{g} / \mathrm{kg}$ daily) and albendazole after Strongyloides was seen in BAL and the stool sample. She also received doxycycline and metronidazole for the presumed $B$. coli infection.

However, the BAL sample was re-evaluated and the diagnosis of B. coli was later revised in favour of ciliocytophthoria as B. coli was never isolated from stool specimens. The hospital course was further complicated by vancomycin-resistant Enterococcus faecium (VRE) bacteraemia, Pseudomonas aeruginosa ventilator-associated pneumonia and relapsing enterococcal (non-VRE) meningitis. The patient died after a prolonged hospital stay.

\section{DISCUSSION}

Strongyloidiasis is caused by the nematode Strongyloides stercoralis. It is endemic in tropical and subtropical areas. In the USA, the Appalachian region has the highest rates of infection ${ }^{[1]}$. The filariform larvae penetrate the skin and migrate through the lungs to the gastrointestinal tract to develop into adult worms. Autoinfection allows persistence of this nematode for several decades in asymptomatic individuals. The cycle of autoinfection can lead to a hyper-infection syndrome in immunocompromised patients. Clinical findings in the hyper-infection syndrome may be attributable to the direct consequences of organ invasion by the filariform larvae and lead to secondary bacteraemia, pneumonia or even meningitis due to bloodstream seeding originating from gastrointestinal tract transmigration. Eosinophilia may be absent. The likelihood of developing the hyper-infection syndrome is increased if cell-mediated immunity is impaired by underlying malignancy, malnutrition, alcoholism, haemopoietic stem cell transplantation, hypogammaglobulinemia or the administration of corticosteroids or cytotoxic drugs ${ }^{[2]}$. Management involves treating the underlying immune deficiency state and use of ivermectin with or without albendazole.

Ciliocytophthoria (CCP) refers to a type of degenerative process observed in the ciliated cells of bronchial epithelium, often associated with viral infections ${ }^{[3]}$, tonsillitis ${ }^{[4]}$ and malignancy ${ }^{[5]}$. Joseph Leidy described the phenomenon in respiratory samples of asthmatic patients in the 19th century. Hilding observed aberrant nasal structures mimicking parasitic cells in $1930{ }^{[6]}$, while Papanicolaou coined the term ciliocytophthoria in $19566^{[5]}$. It has been reported from respiratory ${ }^{[3]}$, gynaecological ${ }^{[7]}$ and peritoneal washings specimens ${ }^{[8]}$ and is seen on fixed, stained cytological specimens as well as fresh preparations. It is often confused with the only known ciliated parasite of humans, $B$. coli. CCP can be differentiated from B. coli infection on the basis of size, distribution of cilia and presence of a nucleus ${ }^{[9]}$. B. coli is a parasite of the large intestine, often found in areas of close human contact with pigs in the background of poor sanitary conditions. The organism is much larger and possesses cilia circumferentially compared with CCP which have a polar distribution of cilia. B. coli often have a kidneyshaped, indented macronucleus, while CCP may be anucleate. High-magnification microscopy and special stains may be used to confirm the diagnosis of CCP.

The existence of CCP as a degenerative process has been corroborated in several in vitro studies using electron microscopy. Recent case reports of isolation of and infection by uncommon protozoa in humans ${ }^{[10]}$ and knowledge of multiflagellated protozoa of the order Hypermastigida inhabiting the alimentary canals of certain insects has raised several questions regarding dismissal of all CCP as a purely degenerative phenomenon.

\section{REFERENCES}

1. Walzer PD, Milder JE, Banwell JG, Kilgore G, Klein M, Parker R. Epidemiologic features of Strongyloides stercoralis infection in endemic areas of the United States. Am J Trop Med Hyg 1982;31:313-319.

2. Seet RCS, Lau LG, Tambyah PA. Strongyloides hyperinfection and hypogammaglobulinemia. Clin Diagn Lab Immunol 2005;12:680-682.

3. Grabowska-Joachimiak A. The morphology of ciliated cells in nasal mucosa during a viral infection. Otolaryngol Pol 1998;52:175-180.

4. Sasaki Y, Abe H, Tokunaga E, Tsuzuki T, Fujioka T. Ciliocytophthoria (CCP) in nasopharyngeal smear from patients with acute tonsillitis. Acta Otolaryngol Suppl 1988;454:175177.

5. Papanicolaou GN. Degenerative changes in ciliated cells exfoliating from the bronchial epithelium as a cytologic criterion in the diagnosis of diseases of the lung. NY State $J$ Med 1956;56:2647-2650.

6. Martínez-Giron R, Doganci L, Ribas A. From the 19th century to the 21st, an old dilemma: ciliocytophthoria, multiflagellated protozoa, or both? Diagn Cytopathol 2008;36:609611.

7. Hollander DH, Gupta PK. Detached ciliary tufts in cervico-vaginal smears. Acta Cytol 1974;18:367-369.

8. Mahoney CA, Sherwood N, Yap EH, Singleton TP, Whitney DJ, Cornbleet PJ. Ciliated cell remnants in peritoneal dialysis fluid. Arch Pathol Lab Med 1993;117:211-213.

9. Hadziyannis E, Yen-Lieberman B, Hall G, Procop GW. Ciliocytophthoria in clinical virology. Arch Pathol Lab Med 2000;124:1220-1223.

10. Wang Y, Tang Z, Ji S, Zhang Z, Chen J, Cheng Z, et al. Pulmonary Lophomonas blattarum infection in patients with kidney allograft transplantation. Transplant Int 2006;19:10061013. 